\title{
Declining extra-pair paternity with laying order associated with initial incubation behavior, but independent of final clutch size in the blue tit
}

\author{
Oscar Vedder • Michael J. L. Magrath • \\ Daphne L. Niehoff • Marco van der Velde • \\ Jan Komdeur
}

Received: 18 October 2011 /Revised: 5 December 2011 / Accepted: 15 December 2011 /Published online: 27 December 2011

(C) The Author(s) 2011. This article is published with open access at Springerlink.com

\begin{abstract}
Although functional explanations for female engagement in extra-pair copulation have been studied extensively in birds, little is known about how extra-pair paternity is linked to other fundamental aspects of avian reproduction. However, recent studies indicate that the occurrence of extra-pair offspring may generally decline with laying order, possibly because stimulation by eggs induces incubation, which may suppress female motivation to acquire extra-pair paternity. Here we tested whether experimental inhibition of incubation during the laying phase, induced by the temporary removal of eggs, resulted in increased extra-pair paternity, in concert with a later cessation of laying, in blue tits (Cyanistes caeruleus). As expected, experimental females showed a more gradual increase in nocturnal incubation duration over the
\end{abstract}

Communicated by S. Pruett-Jones

O. Vedder • M. J. L. Magrath · D. L. Niehoff · M. van der Velde •

J. Komdeur

Behavioural Ecology and Self-Organization Group, Centre for Ecological and Evolutionary Studies, University of Groningen,

P.O. Box 11103, 9700 CC, Groningen, The Netherlands

M. J. L. Magrath

Department of Wildlife Conservation and Science, Zoos Victoria,

Parkville, Victoria 3052, Australia

M. van der Velde

Animal Ecology Group, Centre for Ecological and Evolutionary

Studies, University of Groningen,

P.O. Box 11103, 9700 CC, Groningen, The Netherlands

Present Address:

O. Vedder $(\square)$

Edward Grey Institute, Department of Zoology,

University of Oxford,

South Parks Road,

Oxford OX1 3PS, UK

e-mail: oscar.vedder@zoo.ox.ac.uk laying phase and produced larger clutches than controls. Moreover, incubation duration on the night after the first egg was laid predicted how extra-pair paternity declined with laying order, with less incubation being associated with more extra-pair offspring among the earliest eggs in the clutch. However, incubation duration on this first night was unrelated to our experimental treatment and independent of final clutch size. Consequently, the observed decline in extra-pair paternity with laying order was unaffected by our manipulation and larger clutches included proportionally fewer extra-pair offspring. We suggest that female physiological state prior to laying, associated with incubation at the onset of laying, determines motivation to acquire extra-pair paternity independent of final clutch size. This decline in proportion of extrapair offspring with clutch size may be a general pattern within bird species.

Keywords Brood size $\cdot$ Extra-pair copulation $\cdot$ Fertility insurance $\cdot$ Incubation $\cdot$ Reproductive strategy

\section{Introduction}

Despite the widespread occurrence of extra-pair paternity (EPP) among birds (Griffith et al. 2002), the primary motivation of females to participate in extra-pair copulations (EPCs) that lead to EPP remains contentious and poorly understood (Westneat and Stewart 2003; Arnqvist and Kirkpatrick 2005; Akçay and Roughgarden 2007; Brommer et al. 2007; Griffith 2007). Considerable research has focused on investigating potential functional explanations for female participation in EPCs, such as fertility insurance or the acquisition of good or compatible genes (Sheldon 1994; Jennions and Petrie 2000), yet little is known about how EPP integrates with other aspects 
of avian reproduction. Nevertheless, studies report that extrapair offspring (EPO) are often non-randomly distributed over the laying sequence, with EPO generally being overrepresented in the earlier eggs (house sparrows Passer domesticus; Cordero et al. 1999; collared flycatchers Ficedula albicollis; Krist et al. 2005; blue tits Cyanistes caeruleus; Magrath et al. 2009; house wrens Troglodytes aeon; Johnson et al. 2009; western bluebirds Sialia mexicana; Ferree et al. 2010; but not in: red-winged blackbirds Agelaius phoeniceus; Westneat et al. 1995; tree swallows Tachycineta bicolor; Barber and Robertson 2007). These patterns may be explained by a decreasing female motivation for EPCs after the onset of egg laying (Michl et al. 2002; Magrath et al. 2009; Vedder et al. 2010).

Identifying the mechanism responsible for this decline in EPP with laying order should provide a greater functional understanding of avian reproductive strategies, as this mechanism may shape the relationship between clutch size, the main determinant of avian reproductive effort, and EPP. For example, if EPC activity ceases after laying the first egg but females continue to copulate with their social partner, and fertilization success is determined largely by last male sperm precedence (Birkhead 1998), then the number of EPO would be independent of clutch size causing the proportion of EPO in the clutch to decline with each additional egg that is laid. Alternatively, if the proportion of EPO in the clutch is to remain constant or increase with clutch size, EPC activity would have to cease in concert with the timing of cessation of egg laying. However, to achieve such a connection between temporally disassociated traits, both timing of EPC and clutch completion should share a link to some aspect of the female's physiological state. Recently, we proposed that the underlying physiological state that also determines a female's incubation intensity may provide this link, and cause a concerted timing of motivation for EPC and cessation of egg laying (Vedder et al. 2010).

It is a longstanding idea that incubation during the laying phase predicts cessation of laying (Cole 1930). Haftorn (1981) showed that the progressive increase of nocturnal incubation over the laying phase by great tits (Parus major) was strongly correlated with final clutch size. Since both the amount of incubation and cessation of laying can be altered by manipulating the number of eggs in the nest early during the laying phase (Beukeboom et al. 1988; Hebert and Sealy 1992; Massaro et al. 2007), a common regulatory mechanism underpinning incubation and the cessation of laying appears likely (Meijer et al. 1990; Haywood 1993a; Sockman et al. 2006). This is generally explained by the sensory stimulation of eggs promoting incubation. In turn, ovarian follicular development becomes disrupted once an incubation-related hormonal threshold is reached, resulting in the termination of egg laying after the last mature follicles have been ovulated (Haftorn 1981; Meijer et al. 1990; Haywood 1993a). The few studies that have manipulated egg number and measured incubation behavior in relation to egg laying, support this suggestion as they found that the females that responded to the treatment by increasing or decreasing incubation during the laying phase, produced smaller or larger clutches accordingly (Beukeboom et al. 1988; Meijer 1995). However, it remains unclear whether patterns of incubation in the early laying phase, coinciding with a marked decline in likelihood of EPO in the blue tit laying sequence (Magrath et al. 2009), already predict when egg production will stop.

To date, no study has gathered data on laying order and paternity in combination with incubation over the laying phase. However, an indication that early incubation intensity and EPP covary comes from our previous study on blue tits (Vedder et al. 2010), because females whose clutch hatched more asynchronously after adding model eggs prior to the start of laying, also had a lower proportion of EPO in their nest. We suggested that this pattern was best explained by a decline in female motivation to seek EPC resulting from hormonal changes associated with greater incubation intensity (Vedder et al. 2010), but the absence of incubation data prohibited the testing of this hypothesis. Here we aim to specifically test whether the decline in EPP with laying order in blue tits acts in concert with early incubation intensity and cessation of laying, and thus share a link to a common underlying physiological state.

To address this question, we aimed to experimentally reduce the duration of incubation during laying and increase the number of eggs laid in the clutch. We achieved this by removing up to the first ten eggs on the day of laying while simultaneously monitoring incubation during the entire laying phase. All eggs were numbered in the order of laying and their paternity assessed subsequently. Incubation, clutch size and paternity of these treatment clutches were compared to an unmanipulated control group. If the presence of eggs affects incubation and consequently both clutch size and paternity through a common mechanism, we predict that the experimentally treated females should display reduced incubation during the laying phase, together with larger clutches and a delayed decline in EPP over the laying sequence (and hence more EPO). Alternatively, if EPP and cessation of laying are not linked in concert to laying-phase incubation, we expect that females will respond to the removal of the egg stimulus by laying more eggs but without an increase in the number of EPO, leading to proportionally fewer EPO in larger clutches.

\section{Methods}

Study species and background information

Blue tits are small (10-12 g), cavity-breeding, insectivorous passerines, which typically lay large clutches (8-15 eggs). 
The number of eggs laid can be easily manipulated by removing eggs (one per day) from, or adding eggs to, the nest during the early laying phase (Winkel 1970; Haywood 1993b). Clutches often (about 40\%) contain at least one EPO (e.g., Kempenaers et al. 1997; Magrath et al. 2009), and engagement in EPC is largely under female control (Kempenaers et al. 1992, 1995; Vedder et al. 2010). Females normally lay one egg each day and progressively increase the amount of incubation from around $1 \mathrm{~h}$ at night after the first egg is laid to almost full nocturnal incubation by the end of laying (Haftorn and Reinertsen 1985). In the laying phase, females remain in the nestbox after the nocturnal incubation bout, to roost without heating the eggs, until they lay the new egg in the morning (Haftorn and Reinertsen 1985). Diurnal incubation generally starts around clutch completion, or a few days before (Cramp and Perrins 1993), and incubation is solely performed by the female (Cramp and Perrins 1993).

Study area and general procedures

The study was conducted in the breeding season (April June) of 2009 on 'De Vosbergen' estate near Groningen in the north of the Netherlands $\left(53^{\circ} 08^{\prime} \mathrm{N}, 06^{\circ} 35^{\prime} \mathrm{E}\right)$. The area consists of 54 ha of mixed deciduous and coniferous forest interspersed with areas of open grassland and contains 188 nestboxes designed for blue tits.

All occupied nestboxes were checked daily during the nest building and laying phase from the start of April. Once egg laying started, all nests were randomly assigned to either a control or experimental treatment (see 'Experimental protocol' section), and all new eggs were numbered in the order of laying. At all assigned nests, laying commenced between April 10-19. On the day the first egg was laid, a small temperature logger (iButton [type: DS1921G]; Maxim, Sunnyvale, CA, USA) was placed in the lining underneath the nest-bowl, that recorded temperature (with a resolution of $0.5^{\circ} \mathrm{C}$ ) every $10 \mathrm{~min}$ throughout the laying phase (Fig. 1).

All clutches were collected 11-12 days after either clutch completion or the start of diurnal incubation and replaced with model eggs to prevent desertion. Each egg was placed in an individual compartment within incubators, allowing us to match all hatchlings to the egg from which it hatched (following the protocol described by Magrath et al. 2009). Throughout the day new hatchlings were uniquely marked, by clipping the very tip of one or two toenails in a specific combination, and returned to their nest of origin within $2 \mathrm{~h}$ of hatching. A small blood sample (ca. $10 \mu \mathrm{l}$ ) was collected from the nestlings within 3 days of hatching. Parents were caught inside the nestbox when feeding nestlings 10-12 days after the nestlings hatched. At capture, parents were sexed based on the presence/absence of a brood patch and blood sampled for parentage analyses. Blood samples of parents and hatchling were stored in $100 \%$ ethanol.

\section{Experimental protocol}

As soon as egg laying started, nests were randomly assigned to either a control $(n=41)$ or an experimental
Fig. 1 Typical pattern of temperature variation in the nest-bowl of an unmanipulated female over the course of the laying phase. Laying days on the $x$-axis represent the start of the day (i.e., 00:00 h) on which the respective egg was laid. Shaded areas represent nighttime (i.e., the time between local sunset and sunrise). Consecutive numbers in the graph indicate the small rises in temperature around sunrise, associated with laying the respective egg. Note the increasing duration of periods, starting around sunset, when the temperature is considerably higher than the fluctuations in daily temperature, reflecting female nocturnal incubation (Haftorn and Reinertsen 1985)

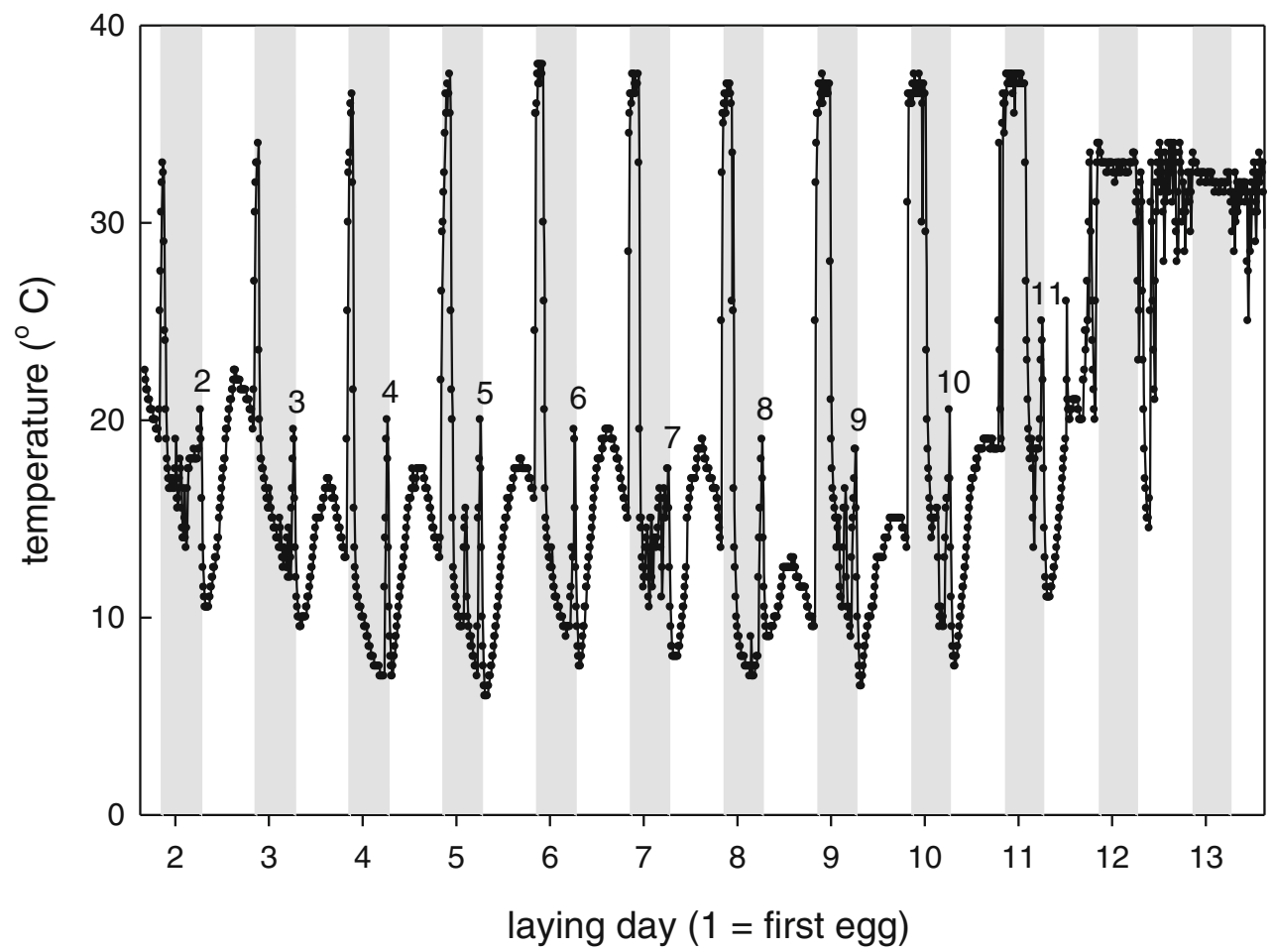


$(n=43)$ treatment. The experimental treatment consisted of collecting the first ten laid eggs, each on the day of laying, such that eggs were never present in the nest at night, when females incubate, for the first 10 days of laying. This method has been applied successfully before to induce increased clutch sizes in blue tits, with relatively little nest abandonment (Haywood 1993b; see also below). Collected eggs were stored at the field site in a room where temperature fluctuated between $10^{\circ} \mathrm{C}$ and $20^{\circ} \mathrm{C}$, generally following the diurnal cycle. The stored eggs were rotated once a day. On the day the 11th egg was laid, one half of the ten temporarily stored eggs (i.e., $1-5$ or 6-10) was selected randomly and returned to the nest, with the remaining half returned the following day. The control group was only subjected to the general procedures, hence these nests were visited equally frequently over the first 10 days of laying, but the eggs were left to accumulate in the nest as normal. We chose to manipulate incubation intensity by egg removal, instead of egg addition prior to laying, because of the difficulty in predicting the onset of laying and the problem of some females failing to accept model eggs (Vedder et al. 2010). We expected that the temporary removal of ten eggs would be sufficient to cause a detectable effect on EPP, if the decline in EPP with laying order is indeed linked to incubation and clutch size.

\section{Quantification of incubation}

A typical example of the incubation data we collected is shown in Fig. 1, exactly corroborating the findings of Haftorn and Reinertsen (1985). Since the peak nightly temperature was highly variable (apparently associated with the distance between the temperature logger and the nest-bowl), but clearly distinguishable from the background ambient temperature, we visually inspected temperature profiles blind to nest treatment. We quantified the duration of nightly incubation bouts by subtracting the time temperature started to rise, relative to gradual fluctuations in ambient temperature, from the time temperature started to decline again. Each nightly bout was quantified until incubation became continuous. Note that the criteria to quantify incubation behavior were independent of treatment. Hence, although there were no eggs present for the first 10 nights of laying in the experimental group, the behavior of heating the nest-bowl, similar to that in the control group where eggs were present, was communally classified as incubation behavior. When functioning as a dependent variable in our analyses, incubation data were log-transformed to attain a normal distribution.
Molecular parentage analyses

DNA was extracted from both parent and nestling blood samples using a chelex extraction method (Walsh et al. 1991). To exclude paternity, parents and nestlings were genotyped for six microsatellite loci: Pca3, Pca7, Pca8 and Pca9 (Dawson et al. 2000), Pocc6 (Bensch et al. 1997) and Pdo5 (Griffith et al. 1999). For details on PCR reactions, see Vedder et al. (2010). Fluorescently labeled PCR products were separated on an AB3730 DNA analyzer and allele lengths determined using Genemapper 4.0 software. Using Cervus 3.0 (Kalinowski et al. 2007), mean exclusion probability of the six markers was calculated to be 0.99934 for the first (female) parent and 0.99997 for the second (male) parent (given the genotype of the first parent). Maternity of the social female was confirmed from the microsatellite data for all nestlings. Paternity of the social male was excluded, and the offspring assigned as extra-pair, if there were at least two mismatches between the genotype of the social father and offspring.

\section{Data and statistical analyses}

After the experiment, we excluded one nest from the control group because it failed after the fourth egg was laid and one nest from the experimental group because the female abandoned the nest after laying the tenth egg. In addition, incubation data were not available for three control and two experimental nests because some females buried the temperature loggers.

For the analyses of EPP, six nests were excluded (two control and four experimental), because they failed before the parents were caught, and an additional two control and four experimental nests because they belonged to single females (i.e., no feeding male present) or secondary females of polygynous males (following Vedder et al. 2010). Of the remaining nests, we blood-sampled and assigned paternity (extra-pair or within-pair) to $98.5 \%(n=778)$ of hatchlings, though egg-specific paternity was not determined for two control nests because hatching started before the anticipated hatching date. At 37 of these nests we permanently collected the second and tenth eggs on the day of laying as part of another study (19 control and 18 experimental nests). Hence, sample sizes vary between analyses.

Analyses of dependent variables that varied only at the nest level (e.g., clutch size, occurrence of EPP in the nest) were performed in SPSS 16.0. Binary variables were always analyzed with logistic regression. Analyses of hatching probability, nocturnal incubation duration and individual nestling paternity were conducted using hierarchical random intercept models in MLwiN 2.02 (Rasbash et al. 2004), to account for the non-independence and hierarchical structure of the data. These models included a random component 
with nest identity at level two and laying day/nestling identity at level one. To test for effects on the binary responses of hatching probability and nestling paternity (within-pair vs. extra-pair), we used a binomial error distribution with a logit link function. The significance (two-tailed) of the explanatory terms was determined using the Wald statistic, which approximates the $\chi^{2}$ distribution. Testing was hypothesisbased, and final models where multiple explanatory variables were tested simultaneously were derived through backwards elimination of least significant terms, starting with interaction terms. Only terms with $P<0.05$ were included in the final model. For all analyses, significance and statistics of interaction terms were presented with single terms included in the model.

Ethical standards

All procedures involving handling of blue tits were permitted by the Animal Experiments Committee of the University of Groningen and comply with current Dutch law.

\section{Results}

Overall hatching success (excluding nests that failed completely before hatching) was not significantly different between the groups (control: $92.9 \%, n=439$, experimental: $\left.88.9 \%, n=524, \chi^{2}=2.66, d f=1, P=0.10\right)$. However, the temporary storage of eggs in the experimental group might have had some adverse affect on hatchability, as the difference was significant (control: $93.0 \%, n=344$; experimental: $\left.86.0 \%, n=349 ; \chi^{2}=4.88, d f=1, P=0.027\right)$ when only the first ten eggs were compared. Moreover, the interaction effect between laying order and experimental treatment on hatchability among the first ten eggs was almost significant (coefficient $\pm \mathrm{SE}=0.19 \pm 0.10, \chi^{2}=3.70, d f=1, P=0.054$ ), suggesting that early laid eggs in the experimental group, which were stored the longest, were least likely to hatch. A reduced hatchability of early eggs in the experimental group would (all else equal) lead to reduced numbers of EPO in this group, since EPO are more common among early eggs (Magrath et al. 2009, see also below). To circumvent this potential effect, we included laying order as a covariate in the analyses on EPP, and so analysed paternity at the level of the individual hatchling.

Incubation and the number of eggs laid

Our manipulation had a highly significant effect on the total number of eggs laid, as the experimental females laid an average of 2.1 more eggs than the control females (mean \pm SE; control: $12.4 \pm 0.19, n=40$; experimental: $14.5 \pm 0.27$, $n=42 ; t=-6.20, \mathrm{df}=80, P<0.001)$. The progressive increase in duration of nocturnal incubation over the laying phase was also significantly delayed among the experimental females, as indicated by a significant treatment by laying day interaction effect on incubation duration (Fig. 2a; $n=37$ control and 40 experimental nests, $\chi^{2}=63.44, d f=1, P<$ $0.001)$. However, despite the fact that no eggs were present in manipulated nests for the first 10 nights of laying, all females spent at least some time incubating every night during the laying phase.

Closer examination of the incubation data revealed that between female variation in the increase of incubation duration over the laying phase can be explained by the total number of eggs a female laid, as evident from the significant interaction effect between laying day and the total number of eggs laid on incubation duration (Fig. 2b; Table 1). This

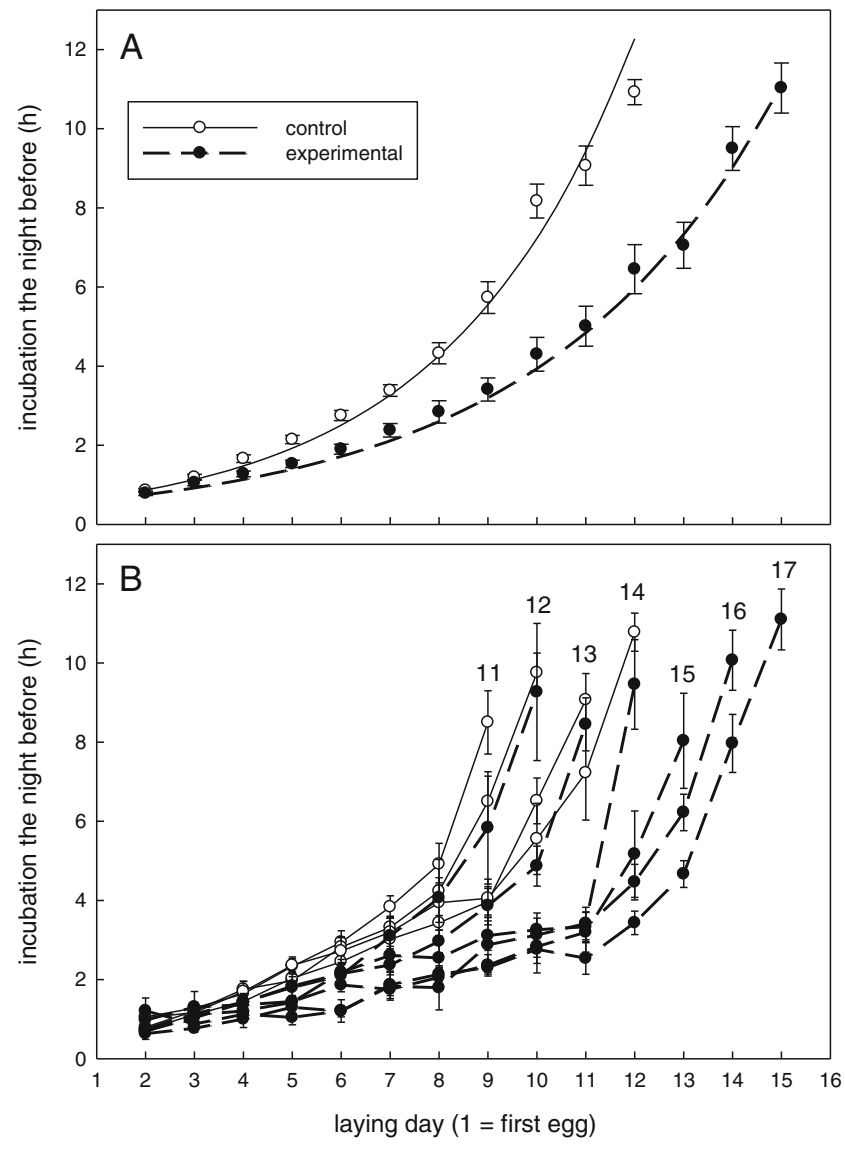

Fig. 2 Average $( \pm \mathrm{SE})$ duration of nocturnal incubation before the day of laying the respective egg. a Data subdivided into treatment group with trend lines representing model predictions. Initial sample sizes for the control and experimental group were 37 and 40 nests, respectively. b Control and experimental groups subcategorized into groups of females that laid the same total number of eggs with the lines connecting the values in each subcategory. The numbers in the graph, above final values, indicate the total number of eggs laid in that category. Sample sizes for control categories 11,12, 13 and 14 are 6,13,10 and 6 nests, respectively. Sample sizes for experimental categories 12, 13, 14, 15, 16 and 17 are 5, 10, 4, 6, 9 and 5 nests, respectively. Categories are only depicted when multiple females laid the same total number of eggs 
Table 1 Model summary of the effects of experimental treatment, laying day and the total number of eggs laid on the duration of nocturnal incubation during the laying phase

The final model was obtained by stepwise removal of least significant terms from the full model. Statistics for non-significant terms were derived by entry back into the final model

\begin{tabular}{lrrrr}
\hline Explanatory variable & Coefficient (SE) & \multicolumn{1}{c}{$\chi^{2}$} & $\Delta d f$ & $P$ \\
\hline Laying day & $0.237(0.012)$ & 422.64 & 1 & $<0.001$ \\
No. of eggs laid & $0.024(0.009)$ & 6.80 & 1 & 0.009 \\
Treatment (control = ref) & $-0.101(0.025)$ & 16.06 & 1 & $<0.001$ \\
Laying day $\times$ no. of eggs laid & $-0.010(0.001)$ & 19.25 & 1 & $<0.001$ \\
Non-significant & $-0.005(0.004)$ & 1.50 & 1 & 0.221 \\
Treatment $\times$ laying day & $-0.010(0.016)$ & 0.39 & 1 & 0.532 \\
Treatment $\times$ no. of eggs laid & $0.004(0.003)$ & 2.15 & 1 & 0.143 \\
Treatment $\times$ laying day $\times$ no. of eggs laid & &
\end{tabular}

pattern did not differ between the two treatment groups (no interaction effect of laying day, no. of eggs laid and treatment; Table 1). However, experimental females generally incubated less than expected based on the total number of eggs they laid (negative effect of treatment; Table 1). This earlier cessation of laying by experimental females than predicted from the incubation profile over the laying period, compared to the controls, is most likely explained by a rapid cessation of egg production in response to the return of removed eggs on the 11th and 12th laying days (Fig. 2b).

To assess whether the duration of nocturnal incubation in the early laying phase is a good predictor of the final clutch size under natural circumstances, we calculated the $R^{2}$ value for the negative correlation between incubation duration and the number of eggs laid by control females for each laying day separately. This value became higher than 0.10 only from the eighth laying day onwards $\left(R^{2}=0.11, n=37, P=\right.$ 0.040 ), reaching a maximum of 0.42 on the night before the tenth laying day $(n=35, P<0.001)$. Hence, the predictive power of nocturnal incubation becomes substantial only close to the cessation of egg laying.

Extra-pair paternity and the number of eggs laid

Within all broods combined, there was a strong decrease in likelihood of EPP with laying order (Fig. 3; Table 2A). The experimental treatment of egg removal had no effect on an individual hatchling's likelihood of being extra-pair (Fig. 3; Table 2A), nor did the likelihood of EPP decline differently in the experimental groups (Fig. 3; Table 2A). When only including broods with mixed paternity in the analysis, the likelihood of EPP also decreased rapidly with laying order (Table 2B), with no additional effect of treatment (Table 2B) or a treatment $\times$ laying order interaction (Table $2 \mathrm{~B}$ ).

This implies that females laying more eggs should have broods with proportionally fewer EPO. Among all broods, there was indeed a negative relationship between the proportion of EPO and the number of eggs laid (coefficient \pm
Fig. 3 Average ( \pm binomial SE) proportion of extra-pair offspring in relation to position in the laying order, for control and experimentally manipulated clutches. The trend line represents the model prediction. Sample size for each position in the laying order is shown for both control $\left(n_{\mathrm{c}}\right)$ and experimental $\left(n_{\mathrm{e}}\right)$ groups

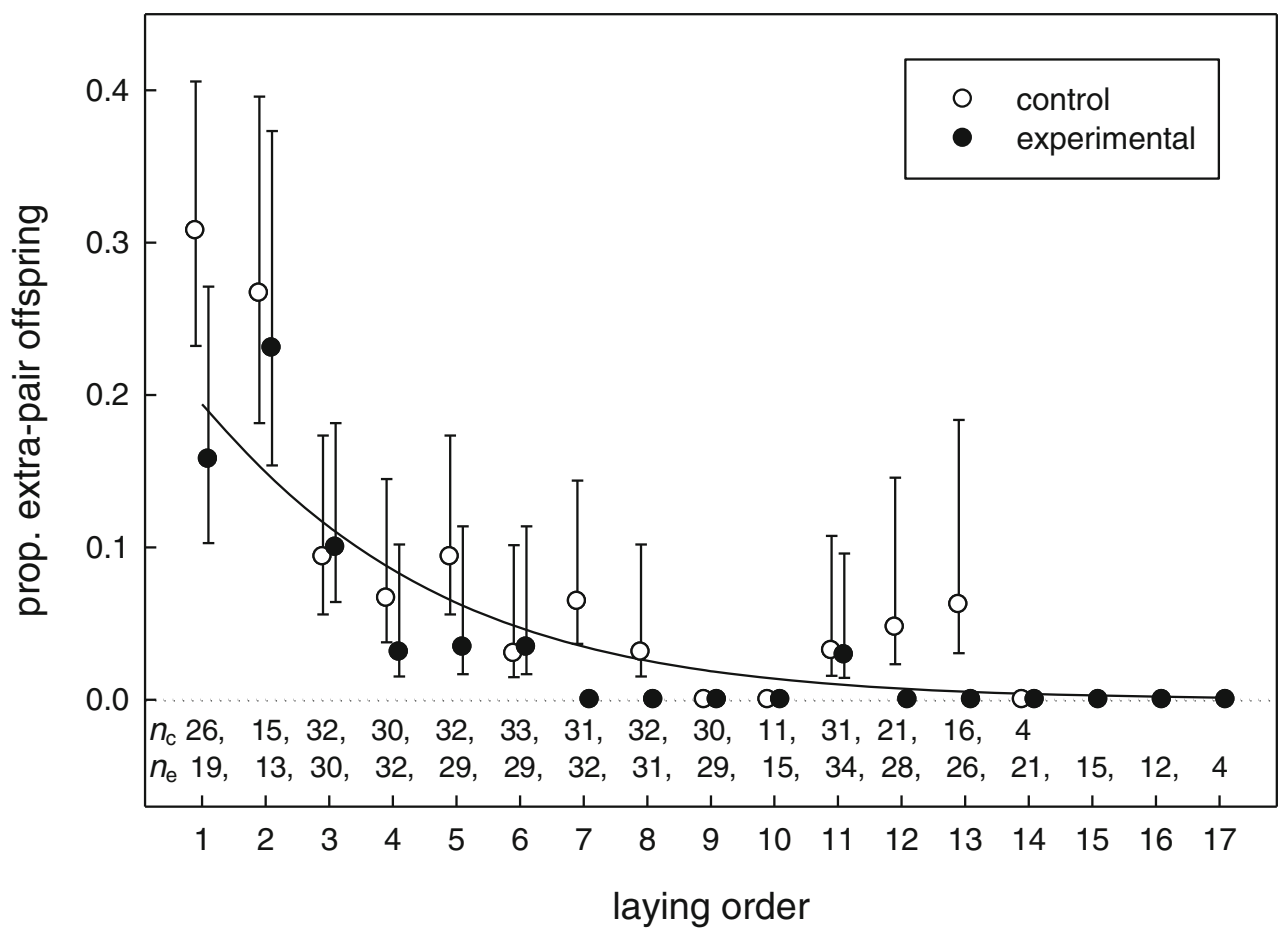


Table 2 Model summary of the effect of the experimental treatment, and laying order, on the probability that a hatchling was sired by an extra-pair male, for (A) all broods and (B) only broods with mixed paternity

\begin{tabular}{lllll}
\hline Explanatory variable & $\begin{array}{l}\text { Coefficient } \\
\text { (SE) }\end{array}$ & $\chi^{2}$ & $\Delta d f$ & $P$ \\
\hline $\begin{array}{l}\text { A. All broods } \\
\text { Laying order } \\
\text { Non-significant }\end{array}$ & $-0.32(0.06)$ & 26.96 & 1 & $<0.001$ \\
$\begin{array}{l}\text { Treatment } \\
\quad \text { control = ref) }\end{array}$ & $-0.71(0.47)$ & 2.29 & 1 & 0.130 \\
$\begin{array}{l}\text { Treatment } \times \text { laying } \\
\text { order }\end{array}$ & $-0.17(0.14)$ & 1.39 & 1 & 0.238 \\
$\begin{array}{l}\text { B. Broods with mixed paternity only } \\
\text { Laying order } \\
\text { Non-significant }\end{array}$ & $-0.35(0.07)$ & 28.13 & 1 & $<0.001$ \\
$\begin{array}{l}\text { Treatment } \\
\text { (control = ref) }\end{array}$ & $0.12(0.41)$ & 0.09 & 1 & 0.764 \\
$\begin{array}{l}\text { Treatment } \times \text { laying } \\
\text { order }\end{array}$ & $0.23(0.17)$ & 1.78 & 1 & 0.182 \\
\hline
\end{tabular}

Final models were obtained by stepwise removal of least significant terms from the full model. Statistics for non-significant terms were derived by entry back into the final model.

$\mathrm{SE}=-0.22 \pm 0.09, \chi^{2}=5.93, d f=1, P=0.015$ ), which was also apparent in mixed paternity broods only (coefficient \pm $\left.\mathrm{SE}=-0.20 \pm 0.09, \chi^{2}=4.95, d f=1, P=0.026\right)$. Both the probability that a brood contained at least one EPO (coefficient \pm $\left.\mathrm{SE}=-0.03 \pm 0.14, \chi^{2}=0.05, d f=1, P=0.83\right)$ and the number of EPO in a brood (Poisson regression: coefficient \pm $\left.\mathrm{SE}=-0.13 \pm 0.09, \chi^{2}=2.20, d f=1, P=0.14\right)$ were independent of the number of eggs laid.
To exclude the possibility that the decline in proportion of EPO with increasing clutch size was only an artifact of the experimental effect on clutch size and hatchability of early eggs, we also examined the relationship between EPP and clutch size in the control group only, combined with all similar data from the same study population over the 3 previous years (for details, see Magrath et al. 2009; Vedder et al. 2010). This confirmed the absence of a relationship between the number of EPO and clutch size (Poisson regression: coefficient $\pm \mathrm{SE}=0.000 \pm 0.034, \chi^{2}=0.00, d f=1$, $P=1.00)$, resulting in a decline in proportion of EPO with clutch size (logistic regression: coefficient $\pm \mathrm{SE}=-0.086 \pm$ $0.041, \chi^{2}=5.13, d f=1, P=0.024 ; n=234$ clutches, 2,367 genotyped offspring).

Extra-pair paternity and incubation

Incubation on the first night after laying start was not affected by the experimental treatment $(n=37$ control and 40 experimental nests, $t=1.05, d f=75, P=0.30$ ), which allowed us to combine all nests to test whether incubation duration at this stage predicted the likelihood of individual hatchlings being sired by an extra-pair male. Together with the effect of laying order (see also above), the likelihood of EPP was indeed strongly related to incubation on the first night (Fig. 4; laying order: $\chi^{2}=23.71, d f=1, P<0.001$; incubation: $\chi^{2}=7.57, d f=1$, $P=0.006$ ). Inclusion of the interaction term indicated that the likelihood of EPP declined more steeply with laying order when females incubated less on the first night (Fig. 4; laying order $\times$ incubation: $\left.\chi^{2}=12.64, d f=1, P<0.001\right)$. Visual
Fig. 4 Average ( \pm binomial SE) proportion of extra-pair offspring in relation to position in the laying order, for females that incubated less and more than average $(0.81 \mathrm{~h})$ on the first night after laying start. The solid trend line represents the model prediction for the average duration of incubation and the dashed lines represent the model predictions for 1 standard deviation $(0.32 \mathrm{~h})$ less and more than average incubation. Sample sizes for each position in the laying order are depicted for both below $\left(n_{-}\right)$ and above $\left(n_{+}\right)$average incubation durations

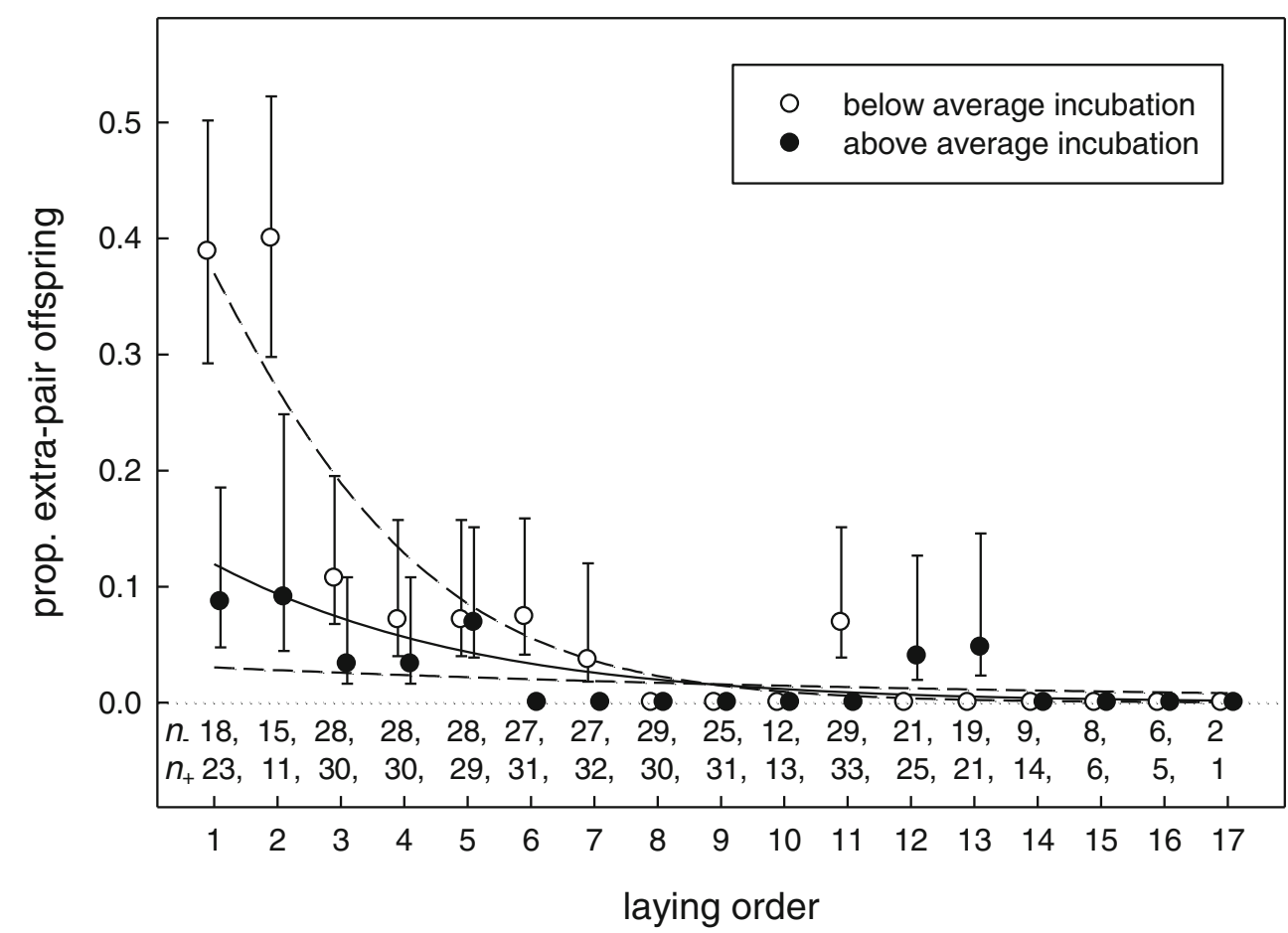


inspection of Fig. 4 suggests that this primarily reflected an increased likelihood of EPP among the first eggs in clutches of females that incubated less (Fig. 4). Nevertheless, when only clutches with mixed paternity were included in the analysis, there was still a highly significant interaction between laying order and first-night incubation on EPP (coefficient $\pm \mathrm{SE}=$ $\left.0.83 \pm 0.24, \chi^{2}=12.13, d f=1, P<0.001\right)$.

To assess whether incubation at a later stage in the laying phase also predicted the pattern of EPP over the laying order, under unmanipulated circumstances, we tested for an interaction effect between laying order and incubation duration on EPP among all control broods, for incubation duration on each night after laying start, separately. As with the analysis for all clutches combined, the interaction was significant on the first night after laying start among control broods only (coefficient $\pm \mathrm{SE}=0.84 \pm 0.30, \chi^{2}=7.90, d f=1, P=0.005$ ). However, this interaction was no longer significant, and the coefficient close to zero, for incubation duration on the night after the second egg was laid (coefficient $\pm \mathrm{SE}=0.05 \pm 0.21$, $\left.\chi^{2}=0.05, d f=1, P=0.82\right)$ and the coefficient remained close to zero over the remainder of the laying period. The fact that only incubation on the first night after egg laying predicted the decline in EPP over the laying order, explains the absence of an experimental effect on EPP (see above), and the poor correlation between incubation on the first and second nights $\left(R^{2}=0.06, n=37, P=0.14\right)$.

\section{Discussion}

Although both the decline of EPP over the laying order and the total number of eggs laid by a female correlated with the duration of nocturnal incubation at some stage of the laying phase, there was no evidence for a concerted response in number of EPO and clutch size to our experimental treatment. Hence, we found no support for the hypothesis that the decline in EPP with laying order acts in concert with early incubation intensity and cessation of laying.

\section{Incubation and extra-pair paternity}

We found that only incubation duration on the first night after the onset of laying correlated with the likelihood of hatchlings being sired by an extra-pair male. This resulted predominantly from an increased likelihood of the first few eggs in the laying order producing EPO, among the clutches of females that incubated less, which makes it unlikely that the level of incubation at this stage causally affected paternity. Since eggs are generally fertilized a day before laying (Etches 1996), paternity of the first two eggs would have already been determined by the first night after laying start, and copulations that fertilize the third egg would most likely have already occurred (Lifjeld et al. 1997). Yet, our previous experiment, where we added model eggs prior to the onset of laying, did affect paternity with a decrease in EPP, and increased hatching asynchrony (Vedder et al. 2010), suggesting that there is an underlying causal link between incubation and paternity. Our current findings suggest that this link is not caused by direct effects of incubation on the acquisition of EPP, but rather reflects some form of physiological trade-off, with the physiological state associated with EPP acquisition prior to laying still influencing a female's incubation duration on the first night after laying start.

Our finding that paternity was not affected by the lack of stimulation by eggs after laying start, may be best explained by females ceasing EPC activity at the onset of laying, regardless of later stimuli that can still affect final clutch size. Future studies attempting to pinpoint specific external stimuli, or the underlying physiological state, that determine individual levels of EPP, may therefore be most promising when targeting the phase prior to laying.

Attributing functional significance to the relationship between earliest incubation intensity and between-female variation in the acquisition of EPP would be highly speculative, considering that one standard deviation in earliest incubation duration is only $0.32 \mathrm{~h}$ (range $=0.17-2.00 \mathrm{~h}$ ), which is unlikely to have any significant consequence for hatching asynchrony. Our findings do show that the advantage enjoyed by EPO of generally hatching earlier than their within-pair half-siblings (Magrath et al. 2009) is not further amplified by females with EPO increasing early incubation to promote greater hatching asynchrony. Nevertheless, the observed correlation between early incubation (a female trait) and the decline of EPP over the laying order does suggest that between-female variation in level of EPP reflects genuine differences in female state, rather than random variation due only to stochastic processes in fertilization success.

\section{Incubation and clutch size}

Consistent with the idea of a shared regulatory mechanism between incubation and clutch size (Meijer et al. 1990; Haywood 1993a; Sockman et al. 2006), we found that females that more gradually increased incubation over the laying phase also laid more eggs, independent of treatment (Fig. 2b). However, experimental females started with the same duration of incubation as controls and even increased the duration of incubation over the laying phase, although on average more slowly, despite the absence of eggs over the first ten nights after laying start. Hence, females may largely follow an endogenous program once laying starts, only partly sensitive to external feedback from the actual number of eggs in the nest. Yet, the females that were sensitive to the absence of eggs clearly showed a correlated response in terms of incubation and clutch completion, leading to larger clutches in concert with a more gradual 
increase of incubation over the laying phase. Such a concerted response may have evolved to optimize the degree of hatching asynchrony, but may limit individual plasticity in adjustment of one trait relative to the other.

\section{Extra-pair paternity and clutch size}

As the presence and number of EPO in a clutch was independent of clutch size, the proportion of EPO declined with the number of eggs laid. This finding was also confirmed in a larger unmanipulated set of clutches that included 3 previous years of data. We suggest that this relationship may be apparent in all species with a declining likelihood of EPO over the laying order, but may be difficult to detect in limited datasets (see Vedder et al. 2010), especially in species with smaller clutch size. A meta-analysis of EPP across bird species failed to reveal a general within-species effect of clutch size on proportion of EPO, but only five out of 65 reviewed studies reported the relationship between paternity and clutch size, and the average sample size in these studies was only 36 clutches (Møller and Ninni 1998). We suggest that future studies should routinely report on the relationship between the proportion of EPO and clutch size to determine if this pattern is general among birds, as it may have important implications for male investment strategies.

Acknowledgements We thank Anna Harts, Peter Wolfs, Marieke Krikke and Annelies Folbert for assistance with the fieldwork. We are also grateful to the 'Kraus-Groeneveld Stichting' for allowing us to work on 'De Vosbergen' estate. Comments by Bart Kempenaers and two anonymous referees greatly improved the manuscript. The research was financially supported by grant numbers 028696 and 043318 to J.K., which are components of the European Community's Sixth Framework Program.

Conflict of interest The authors declare that they have no conflict of interest.

Open Access This article is distributed under the terms of the Creative Commons Attribution Noncommercial License which permits any noncommercial use, distribution, and reproduction in any medium, provided the original author(s) and source are credited.

\section{References}

Akçay E, Roughgarden J (2007) Extra-pair paternity in birds: review of the genetic benefits. Evol Ecol Res 9:855-868

Arnqvist G, Kirkpatrick M (2005) The evolution of infidelity in socially monogamous passerines: the strength of direct and indirect selection on extrapair copulation behavior in females. Am Nat 165:S26-S37

Barber CA, Robertson RJ (2007) Timing of copulations and the pattern of paternity in relation to laying order in tree swallows Tachycineta bicolor. J Avian Biol 38:249-254

Bensch S, Price T, Kohn J (1997) Isolation and characterization of microsatellite loci in a Phylloscopus warbler. Mol Ecol 6:91-92
Beukeboom L, Dijkstra C, Daan S, Meijer T (1988) Seasonality of clutch Size determination in the kestrel Falco tinnunculus - an experimental approach. Ornis Scand 19:41-48

Birkhead TR (1998) Sperm competition in birds. Rev Reprod 3:123-129

Brommer JE, Korsten P, Bouwman KA, Berg ML, Komdeur J (2007) Is extrapair mating random? On the probability distribution of extrapair young in avian broods. Behav Ecol 18:895-904

Cole LJ (1930) The laying cycle in the house wren. Wilson Bull 42:7878

Cordero PJ, Wetton JH, Parkin DT (1999) Within-clutch patterns of egg viability and paternity in the house sparrow. J Avian Biol 30:103-107

Cramp S, Perrins CM (1993) Birds of the Western Palearctic, vol 7. Oxford University Press, Oxford

Dawson DA, Hanotte O, Greig C, Stewart IRK, Burke T (2000) Polymorphic microsatellites in the blue tit Parus caeruleus and their cross-species utility in 20 songbird families. Mol Ecol 9:1941-1944

Etches RJ (1996) Reproduction in poultry. CAB International, Oxford

Ferree ED, Dickinson J, Rendell W, Stern C, Porter S (2010) Hatching order explains an extrapair chick advantage in western bluebirds. Behav Ecol 21:802-807

Griffith SC (2007) The evolution of infidelity in socially monogamous passerines: neglected components of direct and indirect selection. Am Nat 169:274-281

Griffith SC, Stewart IRK, Dawson DA, Owens IPF, Burke T (1999) Contrasting levels of extra-pair paternity in mainland and island populations of the house sparrow (Passer domesticus): is there an 'island effect'? Biol J Linn Soc 68:303-316

Griffith SC, Owens IPF, Thuman KA (2002) Extra pair paternity in birds: a review of interspecific variation and adaptive function. Mol Ecol 11:2195-2212

Haftorn S (1981) Incubation during the egg-laying period in relation to clutch-size and other aspects of reproduction in the great tit Parus major. Ornis Scand 12:169-185

Haftorn S, Reinertsen RE (1985) The effect of temperature and clutch size on the energetic cost of incubation in a free-living blue tit (Parus caeruleus). Auk 102:470-478

Haywood S (1993a) Sensory and hormonal-control of clutch size in birds. Q Rev Biol 68:33-60

Haywood S (1993b) Role of extrinsic factors in the control of clutchsize in the blue tit Parus caeruleus. Ibis 135:79-84

Hebert PN, Sealy SG (1992) Onset of incubation in yellow warblers a test of the hormonal hypothesis. Auk 109:249-255

Jennions MD, Petrie M (2000) Why do females mate multiply? A review of the genetic benefits. Biol Rev 75:21-64

Johnson LS, Brubaker JL, Johnson BGP, Masters BS (2009) Evidence for a maternal effect benefiting extra-pair offspring in a songbird, the house wren Troglodytes aedon. J Avian Biol 40:248-253

Kalinowski ST, Taper ML, Marshall TC (2007) Revising how the computer program CERVUS accommodates genotyping error increases success in paternity assignment. Mol Ecol 16:1099-1106

Kempenaers B, Verheyen GR, Vandenbroeck M, Burke T, Vanbroeckhoven C, Dhondt AA (1992) Extra-pair paternity results from female preference for high-quality males in the blue tit. Nature 357:494-496

Kempenaers B, Verheyen GR, Dhondt AA (1995) Mate guarding and copulation behavior in monogamous and polygynous blue tits do males follow a best-of-a-bad-job strategy. Behav Ecol Sociobiol 36:33-42

Kempenaers B, Verheyen GR, Dhondt AA (1997) Extrapair paternity in the blue tit (Parus caeruleus): female choice, male characteristics, and offspring quality. Behav Ecol 8:481-492

Krist M, Nadvornik P, Uvirova L, Bures S (2005) Paternity covaries with laying and hatching order in the collared flycatcher Ficedula albicollis. Behav Ecol Sociobiol 59:6-11 
Lifjeld JT, Slagsvold T, Ellegren H (1997) Experimental mate switching in pied flycatchers: male copulatory access and fertilization success. Anim Behav 53:1225-1232

Magrath MJL, Vedder O, van der Velde M, Komdeur J (2009) Maternal Effects contribute to the superior performance of extra-pair offspring. Curr Biol 19:792-797

Massaro M, Setiawan AN, Davis LS (2007) Effects of artificial eggs on prolactin secretion, steroid levels, brood patch development, incubation onset and clutch size in the yelloweyed penguin (Megadyptes antipodes). Gen Comp Endocrinol 151:220-229

Meijer T (1995) Importance of tactile and visual stimuli of eggs and nest for termination of egg laying of red junglefowl. Auk 112:483-488

Meijer T, Daan S, Hall M (1990) Family-planning in the kestrel (Falco tinnunculus) - the proximate control of covariation of laying date and clutch size. Behaviour 114:117-136

Michl G, Torok J, Griffith SC, Sheldon BC (2002) Experimental analysis of sperm competition mechanisms in a wild bird population. Proc Natl Acad Sci USA 99:5466-5470

Møller AP, Ninni P (1998) Sperm competition and sexual selection: a meta-analysis of paternity studies of birds. Behav Ecol Sociobiol 43:345-358
Rasbash J, Steele F, Browne W, Prosser B (2004) A user's guide to MLwiN version 2.0. Institute of Education, London

Sheldon BC (1994) Male phenotype, fertility, and the pursuit of extrapair copulations by female birds. Proc R Soc Lond B 257:25-30

Sockman KW, Sharp PJ, Schwabl H (2006) Orchestration of avian reproductive effort: an integration of the ultimate and proximate bases for flexibility in clutch size, incubation behaviour, and yolk androgen deposition. Biol Rev 81:629-666

Vedder O, Magrath MJL, Harts AMF, Schut E, van der Velde M, Komdeur J (2010) Reduced extrapair paternity in response to experimental stimulation of earlier incubation onset in blue tits. Behav Ecol 21:9-15

Walsh PS, Metzger DA, Higuchi R (1991) Chelex-100 as a medium for simple extraction of DNA for PCR-based typing from forensic material. Biotechniques 10:506-513

Westneat DF, Stewart IRK (2003) Extra-pair paternity in birds: causes, correlates, and conflict. Annu Rev Ecol Evol Syst 34:365-396

Westneat DF, Clark AB, Rambo KC (1995) Within brood patterns of paternity and paternal behavior in red-winged blackbirds. Behav Ecol Sociobiol 37:349-356

Winkel W (1970) Experimentelle untersuchingen zur brutbiologie von kohl- und blaumeise (Parus major und P. caeruleus). J Ornithol 111:154-174 